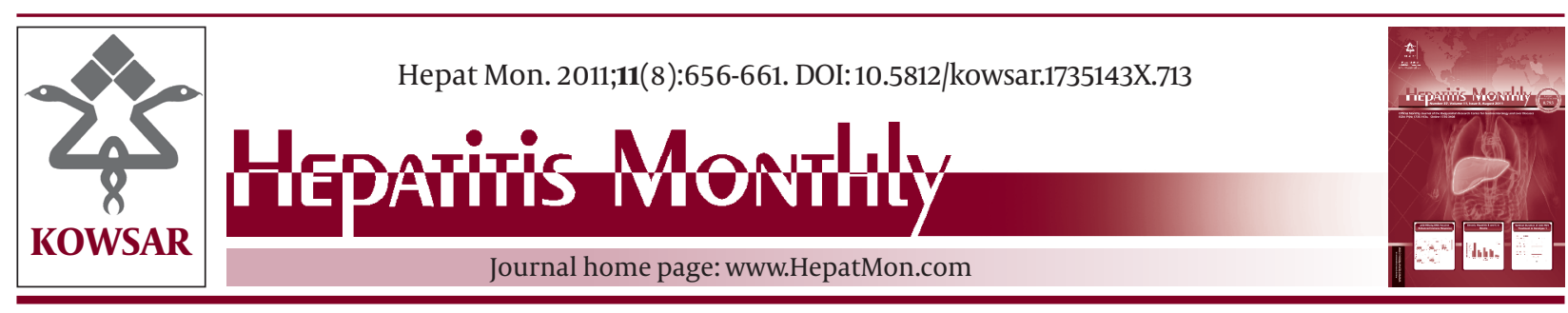

\title{
Liver Injury Induced by High-Dose Methylprednisolone Therapy: A Case Report and Brief Review of the Literature
}

\author{
Krzysztof Gutkowski ${ }^{1^{*}}$, Alina Chwist ${ }^{1}$, Marek Hartleb ${ }^{1}$ \\ ${ }^{1}$ Department of Gastroenterology and Hepatology, Medical University of Silesia, Katowice, Poland
}

\begin{tabular}{ll}
\hline A R T I C L E I N F O & A B S T R A C T \\
\cline { 1 - 1 } $\begin{array}{ll}\text { Article Type: } \\
\text { Case Report }\end{array}$ & $\begin{array}{l}\text { Corticosteroids are used widely to treat many types of disease. In general, these drugs } \\
\text { are considered safe for the liver; however, recent reports have demonstrated that high- } \\
\text { dose methylprednisolone (MT) may cause severe liver injury. Here, we report a case } \\
\text { of a 24-year-old female who was given pulsed MT therapy for multiple sclerosis. MT }\end{array}$ \\
\hline Article history: & $\begin{array}{l}\text { induced icteric hepatitis and impaired liver synthetic function. Hepatotoxicity devel- } \\
\text { oped several weeks after drug exposure, and the causal association with MT was con- } \\
\text { Received:30 May 2011 } \\
\text { Revised:13 Jun 2011 } \\
\text { Accepted:20 Jun 2011 }\end{array}$ \\
\hline
\end{tabular}

Keywords:

Methylprednisolone

Hepatotoxicity

Adverse drug reactions

Multiple sclerosis

๑๑ 2011 Kowsar M.P.Co. All rights reserved.

Implication for health policy/practice/research/medical education:

Corticosteroid-induced liver injury may develop several weeks after short term drug exposure, therefore patients who receive corticosteroid pulses should be screened for potential liver injury.

- Please cite this paper as:

Gutkowski K, Chwist A, Hartleb M. Liver Injury Induced by High-dose Methylprednisolone Therapy: A Case Report and Brief Review of the Literature. Hepat Mon. 2011;11(8):656-61. [DOI:10.5812/kowsar.1735143X.713]

\section{Introduction}

Adverse drug reactions are frequent and remain underestimated causes of acute liver injury that sometimes lead to liver failure, requiring liver transplantation.

High-dose intravenous glucocorticosteroid treatment is one of the most efficient therapeutic options for severe exacerbations of many autoimmune diseases. A review of the literature shows that corticosteroids are not entirely safe for the liver and have been occasionally linked to severe hepatotoxicity (1-3). We present a case

\footnotetext{
${ }^{*}$ Corresponding author at: Krzysztof Gutkowski, Department of Gastroenterology and Hepatology, Medical University of Silesia, ul. Medykow 14, 40752, Katowice, Poland. Tel: +48-327894401, Fax:+48-327894402.

E-mail: kgutski@intertele.pl

DOI: 10.5812/kowsar.1735143X.713

Copyright $\odot$ 2011, BRCGL, Published by Kowsar M.P.Co. All rights reserved.
}

of a 24-year-old woman who was treated with pulsed methylprednisolone (MT) for multiple sclerosis. MT induced serious liver injury, developing as icteric acute hepatitis with impaired prothrombin synthesis. Hepatotoxicity with prolonged latency appeared after the second MT pulse, and the causal relationship between MT and liver injury was confirmed by unintentional rechallenge test.

\section{Case Report}

A 24-year-old woman with a 3-year history of multiple sclerosis and a primary diagnosis of retrobulbar optic neuritis was referred to our department due to severe acute hepatitis. Three months earlier, she was admitted to the neurology department due to exacerbation of multiple sclerosis. Neurological treatment began with 
a 6-day course $(0.5 \mathrm{~g} / \mathrm{d})$ of high-dose intravenous MT, resulting in full recovery of her left arm function. Routine laboratory examinations showed normal liver tests and no autoantibodies in the peripheral blood.

The patient was subsequently switched to beta- $1 \mathrm{~b}$ interferon (Betaferon) treatment and received 2 doses of this drug without any immediate or delayed adverse reactions. After 6 weeks, she developed a subsequent flare of MS, presenting as left-sided limb paralysis. A high-dose intravenous MT pulse (total dose $3.0 \mathrm{~g}$ ) and 2 injections of Betaferon were given, effecting nearly a full recovery. Four weeks after the MT pulse and 1 week after her last Betaferon dose, the patient developed jaundice with elevated serum levels of aspartate aminotransferase (AST) $900 \mathrm{IU} / \mathrm{L}$ (n: 10-31 IU/L), serum alanine aminotransferase (ALT) $1740 \mathrm{IU} / \mathrm{L}$ (n: 9-34 IU/L), serum alkaline phosphatase $186 \mathrm{IU} / \mathrm{L}$ (n:38-126 IU/L), and serum GGTP $50 \mathrm{IU} / \mathrm{L}$ (n:-38 IU/L). The serum bilirubin level was $17.9 \mathrm{mg} / \mathrm{dL}$ (n: 0.3-1.2 mg/dL), the direct bilirubin was $16.1 \mathrm{mg} / \mathrm{dL}$, and the prothrombin index was 35.1\% (INR 2.52). The serum AFP level was $17.18 \mathrm{ng} / \mathrm{mL}(\mathrm{n}:<5 \mathrm{ng} / \mathrm{mL})$. The patient had no history of hepatic disease and denied any use of alcohol. In the previous 6 months, the patient took acetaminophen (used only in a single dose before each Betaferon injection) and oral contraception, which was stopped immediately before the second MT pulse. Serological tests for hepatitis B (including antibodies to $\mathrm{HBC}$ ), hepatitis $\mathrm{C}$, hepatitis $\mathrm{A}$, and infection with cytomegalovirus (CMV) were negative. Smooth muscle antibodies (SMA) were found in high titers (>1:320), and autoantibodies against mitochondria and nuclei were undetected by direct immunofluorescence tests. Her copper urinary excretion was not elevated. An ultrasound abdominal examination showed an intact liver and a normal-sized spleen. No liver biopsy was done due to a low prothrombin level. With a MELD (model for end-stage liver disease) score of 26 , the patient was a potential liver transplantation candidate. Fortunately, her liver function improved spontaneously, and the patient was discharged after 3 weeks with normal aminotransferase levels. Considering the potential hepatotoxicity of interferons, we cautiously suspected the Betaferon of inducing liver injury. Three months later, the patient developed a consecutive flare of multiple sclerosis, for which she received a 6-day intravenous MT pulse (total dose $3.0 \mathrm{~g}$ ) without Betaferon. Her neurological symptoms resolved, but 4 weeks later, she was readmitted to our department with symptoms of acute hepatitis. The serum level of AST was $1488 \mathrm{IU} / \mathrm{L}$, ALT was $1129 \mathrm{IU} / \mathrm{L}$, alkaline phosphatase was $164 \mathrm{IU} / \mathrm{L}, \mathrm{GGTP}$ was $168 \mathrm{IU} / \mathrm{L}$, prothrombin index was 47\% (INR 1.71), and serum bilirubin was $7.3 \mathrm{mg} / \mathrm{dL}$. No drugs, as before, were used to treat the liver disease, and after several weeks, aminotransferases and bilirubin reached reference levels.

\section{Discussion}

We report two serious liver injuries that were related to pulsed MT treatment. The diagnosis was confirmed by the dechallenge-rechallenge relationship between drug administration and hepatitis. Based on a literature review, we identified 12 case reports of corticosteroidinduced hepatotoxicity (Table). The clinical course of liver injuryvaried from asymptomatic hypertransaminasemia to fulminant hepatic failure (3 deaths). In vivo liver histopathology was performed in only 8 cases, showing a large spectrum of lesions. Fernandez et al. reported recurrent acute hepatitis that was characterized by necrosis on histopathological examination, related to intravenous MT that was given on 3 occasions for the management of relapsing multiple sclerosis (4). Loraschi et al. reported 2 cases of liver damage that was related to high-dose MT therapy for demyelinating disease (5). The first patient, a 33-year-old man, developed a histologically recognized acute steatohepatitis 5 weeks after last exposure to MT. The second patient, a 27-yearold woman, presented with moderate and asymptomatic augmentation of liver enzymes 6 days after withdrawal of MT. Hypersensitivity reactions were not observed in any patient. Despite their anti-inflammatory and antiallergic properties, corticosteroids also trigger immunoallergic liver injuries. Das et al. reported recurrent liver injuries that occurred 6 weeks and 3 weeks following the second and third course of intravenous MT, respectively, for multiple sclerosis (6). Liver biopsy showed lobular, primarily perivenular, infiltration with activated lymphocytes, eosinophils, and plasma cells. Moreover, Japanese authors reported the occurrence of autoimmune hepatitis, confirmed by liver histology, in a patient with multiple sclerosis who was treated with MP pulses (7). In their opinion, autoimmune hepatitis was a consquence of an immune rebound phenomenon after pulsed MP.

The mechanisms of corticosteroid-induced liver injury are unclear and only occasionally are related to reactivation of $\mathrm{HBV}$ infection or to the excipient of the MT preparation $(6,8,9)$. Though low doses of corticosteroids are considered safe for the liver, chronic administration of these drugs may be associated with steatosis or steatohepatitis $(2,10)$. Intrinsic hepatotoxicity of high doses of corticosteroids is rather unlikely, as serious hepatic injuries that are related to MT occur rarely and are unpredictable. The majority of hepatotoxic drugs causes idiosyncratic reactions. There are two types of idiosyncrasy: immunoallergic and nonallergic (metabolic). The essence of an immunoallergic reaction is a complex interaction between a parent drug or its metabolites with immunologically competent cells, leading to necrosis and apoptosis of hepatocytes. Released cytokines additionally damage liver cells or have immune-modulating effects (11). In metabolic idiosyncrasy, the liver injury is caused by aberrant hepatic metabolism, leading to overproduction of reactive metabolites from a parent compound. In general, idiosyncratic drug-induced hepatotoxicity is considered to be unpredictable and dose-independent (11); however, 


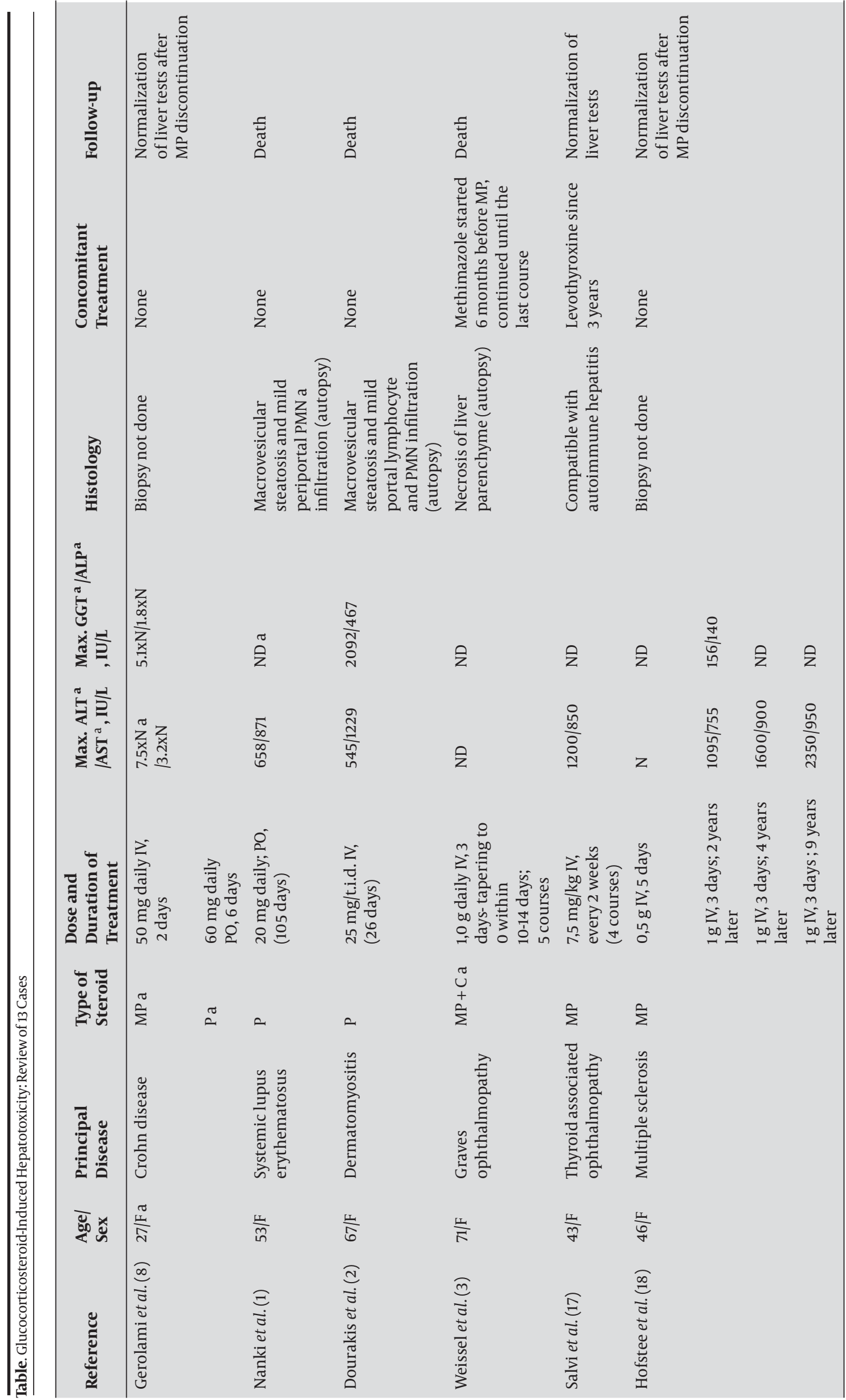




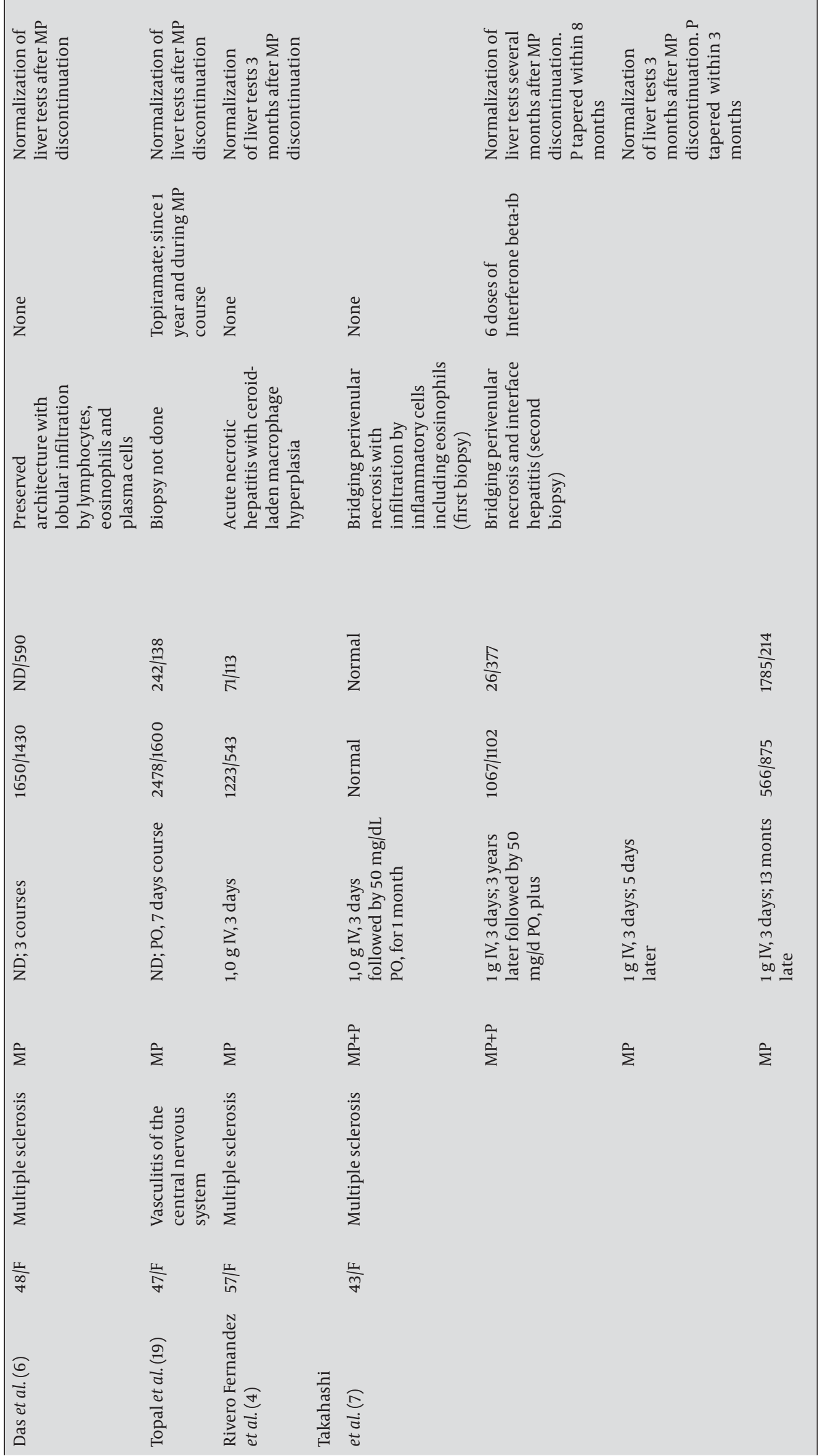




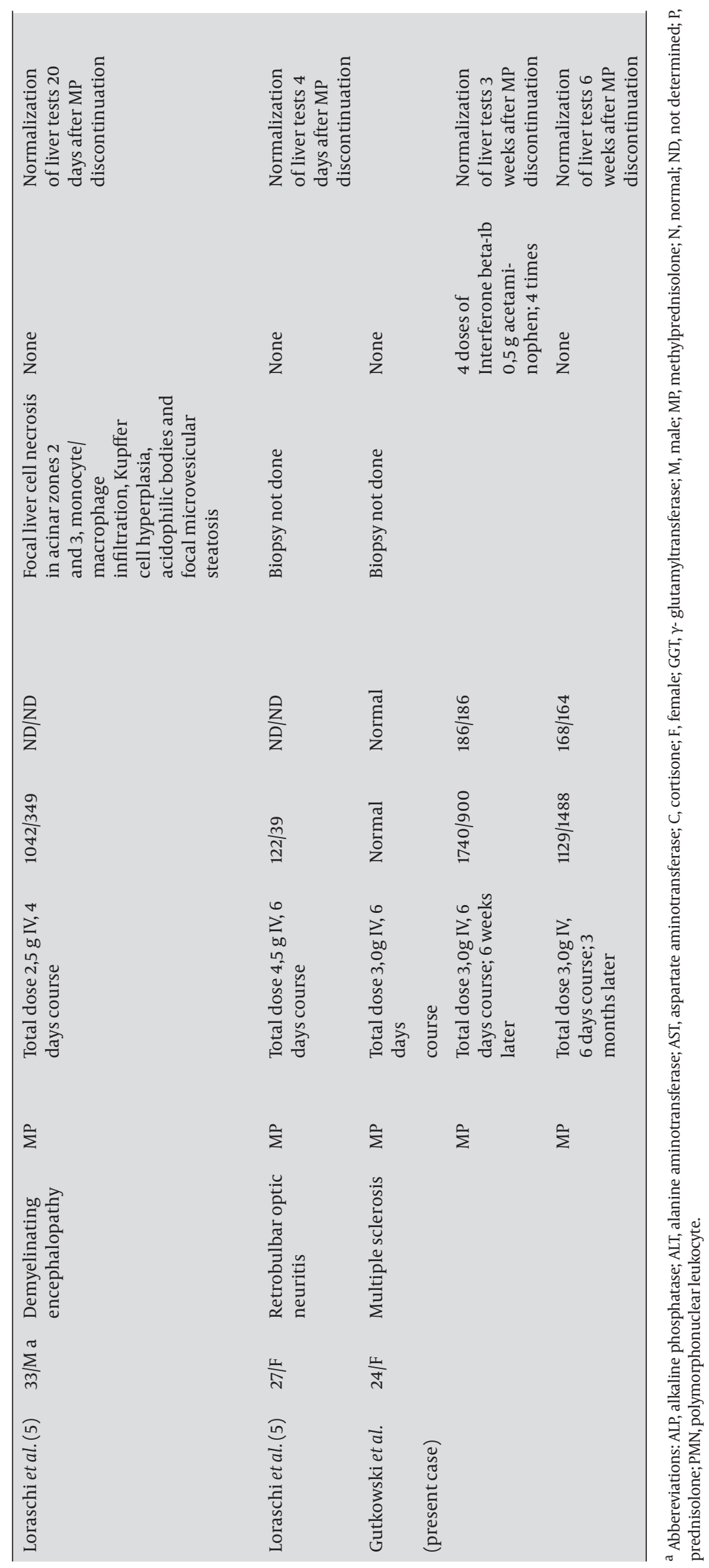


it has been suggested that there is dose-dependency for drugs that are extensively metabolized in the liver (12). MT is metabolized by cytochrome P450 3A4 (CYP3A4), and its metabolites undergo renal elimination.

In our patient, we did not observe any systemic hypersensitivity symptoms before or during liver injury. After two exposures to MT, the hepatitis episodes appeared 4 weeks after drug withdrawal. In metabolic idiosyncrasy, the latency periods vary considerably from days to months, and adverse reactions can occur even several weeks after drug discontinuation. Such examples are not common but have been reported for some antibiotics, such as amoxicillin clavulanate, midecamycin, trovafloxacin, and flucloxacilline (13-16). The duration of anti-inflammatory effects after a single intramuscular injection of 40-80 mg MT ranges from 4 days to 8 days. Thus, it seems unlikely that chemically active metabolites damage hepatocytes 4 weeks after drug discontinuation. Rather, this scenario suggests a delayed immune response to the metabolite that is bound to the host protein and successive presentation as a neoantigen to the immune cells following the death of hepatocytes. The appearance of SMA following exposure to MT might be a feature of the immunological response.

\section{Conclusion}

MT pulses are increasingly used by neurologists, rheumatologists, and endocrinologists to treat various autoimmune diseases. The general awareness of the potential hepatotoxicity of high-dose corticosteroids is very low. Corticosteroid-induced liver injury may occur as acute hepatitis that develops several weeks after shortterm drug exposure. We therefore feel that MT should be placed on the list of hepatotoxic drugs and that patients who receive corticosteroid pulses should be screened for potential liver injury.

\section{Acknowledgements}

None declared.

\section{Financial Disclosures}

None declared.

\section{Funding/Support}

None declared.

\section{References}

1. Nanki T, Koike R, Miyasaka N. Subacute severe steatohepatitis during prednisolone therapy for systemic lupus erythematosis. Am J Gastroenterol.1999;94(11):3379.

2. Dourakis SP, Sevastianos VA, Kaliopi P. Acute severe steatohepatitis related to prednisolone therapy. Am J Gastroenterol. 2002;97(4):1074-5.

3. Weissel M, Hauff W. Fatal liver failure after high-dose glucocorticoid pulse therapy in a patient with severe thyroid eye disease. Thyroid. 2000;10(6):521.

4. Rivero Fernandez M, Riesco JM, Moreira VF, Moreno A, Lopez San Roman A, Arranz G, et al. Recurrent acute liver toxicity from intravenous methylprednisolone. Rev Esp Enferm Dig. 2008;100(11):720-3.

5. Loraschi A, Banfi P, Mauri M, Sessa F, Bono G, Cosentino M. Hepatotoxicity after high-dose methylprednisolone for demyelinating disease. Clin Neuropharmacol. 2010;33(1):52-4.

6. Das D, Graham I, Rose J. Recurrent acute hepatitis in patient receiving pulsed methylprednisolone for multiple sclerosis. Indian J Gastroenterol. 2006;25(6):314-6.

7. Takahashi A, Kanno Y, Takahashi Y, Sakamoto N, Monoe K, Saito H, et al. Development of autoimmune hepatitis type 1 after pulsed methylprednisolone therapy for multiple sclerosis: a case report. World J Gastroenterol. 2008;14(35):5474-7.

8. Gerolami R, Mambrini P, Barthet M, Jean-Pastor MJ, Salducci J, Grimaud JC. Acute hepatitis caused by Solupred in a patient with Crohn disease. Gastroenterol Clin Biol.1997;21(3):236-7.

9. Hammond A, Ramersdorfer C, Palitzsch KD, Scholmerich J, Lock G. Fatal liver failure after corticosteroid treatment of a hepatitis B virus carrier. Dtsch Med Wochenschr. 1999;124(22):687-90.

10. Stravitz RT, Sanyal AJ. Drug-induced steatohepatitis. Clin Liver Dis. 2003;7(2):435-51.

11. Gunawan BK, Kaplowitz N. Mechanisms of drug-induced liver disease. Clin Liver Dis. 2007;11(3):459-75, v.

12. Kaplowitz N. Idiosyncratic drug hepatotoxicity. Nat Rev Drug Discov. 2005;4(6):489-99.

13. Andrade RJ, Lucena MI, Fernandez MC, Vega JL, Camargo R. Hepatotoxicity in patients with cirrhosis, an often unrecognized problem: lessons from a fatal case related to amoxicillin/clavulanic acid. Dig Dis Sci. 2001;46(7):1416-9.

14. Perez Moreno JM, Saldana Gonzalez FJ, Puertas Montenegro M, Baez Perea J. Cholestatic hepatitis caused by midecamycin. Gastroenterol Hepatol.1996;19(9):459-61.

15. Lucena MI, Andrade RJ, Rodrigo L, Salmeron J, Alvarez A, LopezGarrido MJ, et al. Trovafloxacin-induced acute hepatitis. Clin Infect Dis. 2000;30(2):400-1.

16. Olsson R, Wiholm BE, Sand C, Zettergren L, Hultcrantz R, Myrhed M. Liver damage from flucloxacillin, cloxacillin and dicloxacillin. $J$ hepatol.1992;15(1-2):154-61.

17. Salvi M, Vannucchi G, Sbrozzi F, Del Castello AB, Carnevali A, Fargion $\mathrm{S}$, et al. Onset of autoimmune hepatitis during intravenous steroid therapy for thyroid-associated ophthalmopathy in a patient with Hashimoto's thyroiditis: case report. Thyroid. 2004;14(8):631-4.

18. Hofstee HM, Nanayakkara PW, Stehouwer CD. Acute hepatitis related to prednisolone. Eur J Intern Med. 2005;16(3):209-10.

19. Topal F, Ozaslan E, Akbulut S, Kucukazman M, Yuksel O, Altiparmak E. Methylprednisolone-induced toxic hepatitis. Ann Pharmacother. 2006;40(10):1868-71. 